\title{
Secrecy Outage of Max-Min TAS Scheme in MIMO-NOMA Systems
}

\author{
Hongjiang Lei, Member, IEEE, Jianming Zhang, Ki-Hong Park, Member, IEEE, Peng Xu, Zufan Zhang, \\ Gaofeng Pan, Member, IEEE, and Mohamed-Slim Alouini, Fellow, IEEE
}

\begin{abstract}
This paper considers a secure non-orthogonal multiple access system, where confidential messages are transmitted from a base station to multiple legitimate destinations and wiretapped by multiple illegitimate receivers. It is assumed that all the channels experience Nakagami- $m$ fading model and all the nodes are equipped with multiple antennas, respectively. Both non-colluding and colluding eavesdroppers are respectively considered. Max-min (MM) transmit antenna selection (TAS) strategy is adopted to improve the secrecy performance of the target system, in which both users in user paring are considered simultaneously. In particular, closed-form expressions for the cumulative distribution function of the signal-to-interferencenoise ratio at the legitimate user are derived firstly. Then we obtain the exact and asymptotic analytical results in a closed form for the secrecy outage probability of MM TAS scheme. Monte-Carlo simulation results are presented to corroborate the correctness of the analysis. The results show that the secrecy diversity order is zero and non-zero for fixed and dynamic power allocations, respectively.
\end{abstract}

Index Terms-Non-orthogonal multiple access, multiple-input multiple-output, secrecy outage probability, transmit antenna selection.

\section{INTRODUCTION}

\section{A. Background and Related Works}

In last several decades, many technology, such as cooperative communication[1], cognitive radio [2], etc., were proposed to improve the availability of wireless communications. Due to high spectral efficiency, non-orthogonal multiple

Manuscript received.

This work was supported in part by the National Natural Science Foundation of China (NSFC) under Grant 61471076 and 61701066, Chinese Scholarship Council under Grant 201607845004, the Program for Changjiang Scholars and Innovative Research Team in University under Grant IRT_16R72, the special fund for Key Lab of Chongqing Municipal Education Commission, the Project of Fundamental and Frontier Research Plan of Chongqing under Grant cstc2017jcyjAX0204 and cstc2015jcyjBX0085, and the Scientific and Technological Research Program of Chongqing Municipal Education Commission under Grant KJ1600413 and KJ1704088.

H. Lei is with Chongqing Key Lab of Mobile Communications Technology, Chongqing University of Posts and Telecommunications, Chongqing 400065, China. H. Lei is also with CEMSE Division, King Abdullah University of Science and Technology (KAUST), Thuwal 23955-6900, Saudi Arabia (email: leihj@cqupt.edu.cn).

J. Zhang, P. Xu, and Z. Zhang are with Chongqing Key Lab of Mobile Communications Technology, Chongqing University of Posts and Telecommunications, Chongqing 400065, China. (e-mail: cquptzjm@gmail.com, xupeng@cqupt.edu.cn, zhangzf@cqupt.edu.cn).

G. Pan is with Chongqing Key Laboratory of Nonlinear Circuits and Intelligent Information Processing, Southwest University, Chongqing 400715, China (e-mail: gfpan@swu.edu.cn).

K.-H. Park and M.-S. Alouini are with CEMSE Division, King Abdullah University of Science and Technology (KAUST), Thuwal 23955-6900, Saudi Arabia (e-mail: kihong.park@kaust.edu.sa, slim.alouini@kaust.edu.sa). access (NOMA) is accepted as a promising band-efficient candidate technology for the future mobile networks [3] [7]. In NOMA systems, superposition coding is applied at source nodes and successive interference cancellation technology is adopted at destinations. The signals for different users with different channel gains are allocated with different transmission power, respectively. Thus the communications between the base station and multiple users can be proceeded in the same time/frequecy/code simultaneously. The authors of [8] analyzed the outage probability (OP) and ergodic sum rate of a downlink NOMA system and their results demonstrated that NOMA technology can obtain significantly superior performance than the orthogonal multiple access (OMA). The authors of [9] investigated the performance of a downlink NOMA system at a fairness standpoint and the results show that NOMA systems can guarantee high fairness requirements with appropriate power allocation (PA) scheme. The performance of a underlay NOMA large-scale cognitive radio networks was investigated in [10] and the closed-form expressions of $\mathrm{OP}$ were derived using stochastic geometry. The performance of an amplify-and-forward (AF) relaying NOMA system over Nakagami- $m$ fading channels was studied in [11] and the closed-form expressions for the exact and bound of OP and ergodic sum rate were derived.

Multiple-antenna technology has been testified as an efficient scheme to improve the performance of NOMA systems [12]-[14]. The ergodic capacity for multiple-input multipleoutput (MIMO) NOMA systems was studied in [15], [16] and the capacity superiority of MIMO-NOMA over MIMOOMA was proved. Moreover, transmit antenna selection (TAS) scheme has been verified as a low complexity solution to improve performance of NOMA systems when multiple antennas are available at the base station [17]-[22]. The authors of [17] and [18] investigated the performance of an energy harvesting AF relaying NOMA system with TAS scheme and closedform expressions for OP was obtained. A new TAS scheme for multiple-input single-output (MISO) NOMA systems was proposed in [19] and their result testified that higher performance can be obtained with multiple antennas at base station. The authors in [20] proposed an antenna selection algorithm to achieve near-optimal performance and reduce complexity. Two TAS algorithms for MIMO-NOMA systems were proposed and the average sum-rates were analyzed in [21]. The same authors also proposed a joint TAS and PA scheme for a cognitive MIMO-NOMA system and the asymptotic OP was studied in [22].

The openness of wireless channels makes the communi- 
cations become easy and vulnerable to eavesdrop. Physical layer security has been regarded as a promising safeguard technology for wireless communications through utilizing the time-varying nature of the wireless fading channels [23], [24]. In the last several years, the secrecy performance of wireless fading systems was studied in many literatures [25]-[33]. In these great works, the fundamental theories of physical layer security were introduced and the state-of-the-art works on physical layer security technologies were provided. Then the challenges, potential solutions, and future research directions were given, respectively. Many efficient schemes, such as cooperative jamming [34] and interference alignment [35], [36], were proposed to enhance the security of wireless communications.

Nowadays, the secrecy issue of NOMA has been studied in several works [37] - [44]. The security performance of a single-input single-output (SISO) NOMA system was investigated in [37], and the results testified that the secrecy performance of NOMA with optimal PA policy is superior to the one of the conventional OMA. The physical layer security of a downlink NOMA systems was analyzed in [38]. The secure performance of the SISO and MISO NOMA systems was analyzed and both the exact and asymptotic secrecy outage probability (SOP) were studied. The secure performance of cooperative NOMA networks was investigated in [39]. Then, two TAS schemes were proposed for enhancing security performance and the closed-form expressions for the ergodic secrecy rate were obtained. The optimal design of secure NOMA schemes was studied to minimize the transmit power or maximize the minimum confidential information rate subject to the SOP and quality of service constraints in [40]. The physical layer security of a large-scale NOMA network was analyzed in [41], in which all the destinations (both legitimate and illegitimate) are spatially deployed at random locations. Furthermore, the work in [41] was extended in [42] and a MISO NOMA system was considered and artificial noise was employed to heighten the secrecy performance. The practical joint subcarrier assignment and power allocation were firstly proposed by Zhang H. et al. in the secure AF two-way relay NOMA systems with and without cooperative jamming in [43], which can effectively enhance the secrecy energy efficiency. Physical layer security for uplink NOMA scenario was addressed in [44] and the coverage probability and the effective secrecy throughput of each user were studied.

The prior works investigated secure NOMA systems either with a multiple-antenna base station and two single-antenna users [38], [39] or with multiple single-antenna users and a single-antenna source [40]-[44]. Furthermore, all these works focused on the NOMA systems over Rayleigh fading channels. It is more challenging and important to study the secure MIMO-MOMA systems with multiple users over Nakagami- $m$ fading channels due to the following reasons. Firstly, NOMA technology was designed to improve the spectral efficiency, which means that the transmitter can simultaneously send message to multiple (more than two) users. Secondly, as stated before, multiple antennas technology and TAS scheme were proved as an effective method to enhance the security of wireless communication. Finally, as we known, Nakagami- $m$ model describes the wireless fading channel's characteristics more generalized compared with Rayleigh model, which is a special case of Nakagami- $m$ fading.

\section{B. Motivation and Contributions}

To the best of the authors' knowledge, it is still absent to investigate the SOP of MIMO-NOMA systems over Nakagam$m$ fading channels. We list our main contributions of this work as follows.

1) We study the secure communications with a MIMONOMA system. The confidential messages are transmitted from the base station to multiple legitimate destinations and wiretapped by multiple illegitimate receivers. It is assumed that all the channels experience Nakagam- $m$ model and all the nodes are assumed with multiple antennas, respectively. Both non-colluding and colluding eavesdroppers are considered. We propose the max-min (MM) TAS strategy to improve the secure performance, in which both users in user paring are considered simultaneously. The statistical properties of signal-to-interference-noise ratio (SINR) for legitimate nodes are derived firstly. Then we obtain the SOP for MIMO-NOMA systems when MM TAS is utilized at the base station. Monte-Carlo simulations was proceeded to validate the correctness of our results.

2) Furthermore, we analyze the asymptotic secure performance and derive the secrecy diversity order (SDO) for fixed and dynamic PA schemes, which enable a better understanding of the analysis results. The results provide that the SDO with fixed PA is zero and non-zero SDO can be obtained by the dynamic PA scheme.

3) Different from [41] and [42], all the nodes are equipped with multiple antennas and the MM TAS scheme is utilized in our work. Moreover, we consider the Nakagami$m$ fading channel, which is more generalized and accurate wireless model.

4) Compared with [38] and [40], not only multiple legitimate and illegitimate users with multiple antenna are considered in our model, but also a different TAS scheme is considered to improve the secrecy performance. Furthermore, the Nakagami- $m$ fading channel is considered.

The rest of this article is organized as follows. Sec. II introduced the MIMO-NOMA system model and the statistical properties of SINR are given. Sec. III analyzes the SOP of the MM TAS in MIMO-NOMA system. In Section IV, we investigate the asymptotic SOP in MIMO-NOMA system with fixed PA scheme and studied the SDO for the MIMO-NOMA systems with dynamic PA scheme in Section V. The simulation results are resented and discussed in Section VI. Finally, Sec. VII concludes the paper.

\section{SySTEM MODEL}

In this paper, we consider a MIMO-NOMA system, as depicted in Fig. 1, where the base station $(S), M$ legitimate destinations $(D), Q$ eavesdroppers $(E)$ are equipped with $N_{S}\left(N_{S} \geq 1\right), N_{D}\left(N_{D} \geq 1\right)$, and $N_{E}\left(N_{E} \geq 1\right)$ antennas, 


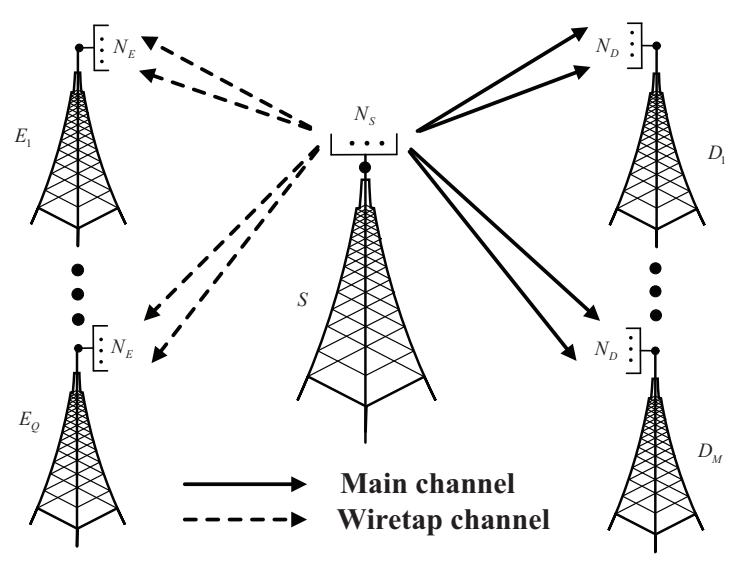

Fig. 1. System model consisting of a base station $(S), M$ legitimate destination nodes $(D)$, and $Q$ illegitimate eavesdropper nodes $(E)$, all equipped with multiple antennas.

respectively. All the main and eavesdropping channels are assumed to undergo quasi-static independent and identically distributed Nakagami- $m$ fading with integer parameters $m_{D}$ and $m_{E}$, respectively. Maximal ratio combining (MRC) is employed at all the legitimate and illegitimate destinations since MRC can maximize the output SNR. We also assume that $m_{D}$ and $m_{E}$ are integer and all the instantaneous channel state information (CSI) is known at $S$.

Similar to [40], [41], [42], it is assumed that all the links between $S$ and $D$ have been ordered as $\sum_{k=1}^{N_{D}}\left|h_{S_{g} D_{1, k}}\right|^{2} \leq$ $\cdots \leq \sum_{k=1}^{N_{D}}\left|h_{S_{g} D_{i, k}}\right|^{2} \leq \cdots \leq \sum_{k=1}^{N_{D}}\left|h_{S_{g} D_{j, k}}\right|^{2} \leq \cdots \leq$ $\sum_{k=1}^{N_{D}}\left|h_{S_{g} D_{M, k}}\right|^{2}$, where $h_{S_{g} D_{i, k}}$ denotes the channel coefficient. It is also assumed that all the users are divided into multiple orthogonal pairs to perform NOMA and each pair is randomly allocated to a specific orthogonal resource slot [41], [42]. For simplicity, a typical pair of users (the $i$-th user and $j$-th user, $i<j$ ) are focused on in our work.

The received instantaneous SINRs of the $i$-th and $j$-th receivers from $g$-th antenna of $S$ are given as [42]

$$
\begin{gathered}
\gamma_{D_{i}}^{g}=\frac{a_{i} \sum_{k=1}^{N_{D}}\left|h_{S_{g} D_{i, k}}\right|^{2}}{a_{j} \sum_{k=1}^{N_{D}}\left|h_{S_{g} D_{i, k}}\right|^{2}+\frac{1}{\rho_{D}}}, \\
\gamma_{D_{j}}^{g}=a_{j} \rho_{D} \sum_{k=1}^{N_{D}}\left|h_{S_{g} D_{j, k}}\right|^{2},
\end{gathered}
$$

respectively, where $\rho_{D}=P_{S} / \sigma_{D}^{2}, P_{S}$ is the transmit power at $S$, and $\sigma_{D}^{2}$ is the noise variance at $D_{k}, a_{l}(l \in\{i, j\})$ represents the PA coefficients, and $a_{i}+a_{j}=1$.

Lemma 1: The cumulative distribution function (CDF) of $\gamma_{D_{i}}$ and $\gamma_{D_{j}}$ can be expressed as

$$
F_{\gamma_{D_{i}}^{g}}(\gamma)=\left\{\begin{array}{ll}
\sum_{k=0}^{M-i} \sum_{\Phi_{i}} A_{D_{i}} e^{-B_{D_{i}} \varphi(\gamma)}(\varphi(\gamma))^{C_{D}}, & \gamma<\frac{a_{i}}{a_{j}} \\
1, & \gamma \geq \frac{a_{i}}{a_{j}}
\end{array},\right.
$$

$$
F_{\gamma_{D_{j}}^{g}}(\gamma)=\sum_{k=0}^{M-j} \sum_{\Phi_{j}} A_{D_{j}}\left(a_{j} \rho_{D}\right)^{-C_{D}} e^{-\frac{B_{D_{j}}}{a_{j} \rho_{D}} \gamma} \gamma^{C_{D}},
$$

$\left\{\left(n_{1}, \cdots, n_{\tau_{D}+1}\right) \in \mathbb{Z}^{\geq} \mid \sum_{p=1}^{\tau_{D}+1} n_{p}=l+k\right\}, \quad \Phi_{l} \geq$ denotes a non-negative integer set, $l \in\{i, j\}, \tau_{D}=m_{D} N_{D}$,

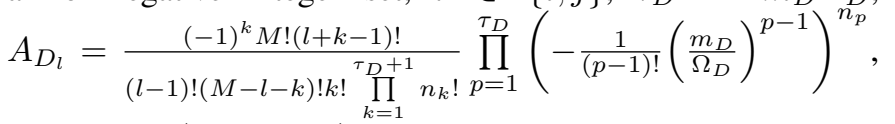
$B_{D_{l}}=\frac{\left(l+k-n_{\tau_{D}+1}\right) m_{D}}{\Omega_{D}}, \quad C_{D}=\sum_{p=1}^{\tau_{D}} n_{p}(p-1)$, and $\varphi(\gamma)=\frac{\gamma}{\rho_{D}\left(a_{i}-a_{j} \gamma\right)}$.

Proof: See Appendix A.

We consider the worst-case case, in which $E_{l}(l=1, \cdots, Q)$ are assumed to have the detection capabilities to detect multiuser data and extract the message to $D_{k}{ }^{1}$. As a result, the SNR at the $v$-th eavesdropper $\left(E_{v}\right)$ which detects the transmitted signal for $D_{l}$ is expressed as

$$
\gamma_{E_{v, l}}^{g}=a_{l} \rho_{E} \sum_{k=1}^{N_{E}}\left|h_{S_{g} E_{v, k}}\right|^{2}, v=\{1, \cdots, Q\},
$$

where $h_{S_{g} E_{v, k}}$ means the channel coefficients, $\rho_{E}=P_{S} / \sigma_{E}^{2}$. To simplify analysis, we assume $\sigma_{D}^{2}=\sigma_{E}^{2}=\sigma^{2}$, then $\rho_{E}=$ $\rho_{D}=\rho$.

In this work, two cases are considered. One is that the eavesdropper works independently, and the other is that they collude. In the scenarios that there is no collusion among all the eavesdroppers, the strongest case is considered. That means

$$
\gamma_{E, l}^{g, \mathrm{~N}}=\max _{v \in\{1, \cdots, Q\}}\left\{\gamma_{E_{v, l}}^{g}\right\} .
$$

If there is a collusion among all the eavesdroppers, we have

$$
\gamma_{E, l}^{g, \mathrm{Y}}=\sum_{v=1}^{Q} \gamma_{E_{v, l}}^{g} .
$$

Using (6), and making use of multinomial theorem [47], we can achieve the probability density function (PDF) of $\gamma_{E}^{g, N}$ as

$$
f_{\gamma_{E, l}^{g, \mathrm{~N}}}(\gamma)=\frac{Q \lambda_{E_{l}} \tau_{E}}{\Gamma\left(\tau_{E}\right)} \sum_{\Phi_{E}} A_{E_{l}} e^{-\left(B_{E_{l}}+\lambda_{E_{l}}\right) \gamma_{\gamma}} \gamma_{E}^{C_{E}+\tau_{E}-1},
$$

where $\Phi_{E}=\left\{\left(n_{1}, \cdots, n_{\tau_{E}+1}\right) \in \mathbb{Z}^{\geq} \mid \sum_{p=1}^{\tau_{E}+1} n_{p}=Q-1\right\}$, $A_{E_{l}}=\frac{(Q-1) !}{\prod_{k=1}^{\tau_{E}+1} n_{k} !} \prod_{p=1}^{\tau_{E}}\left(-\frac{1}{(p-1) !}\left(\lambda_{E_{l}}\right)^{p-1}\right)^{n_{p}}, \quad B_{E_{l}}=$ $\left(Q-1-n_{\tau_{E}+1}\right) \lambda_{E_{l}}, \quad C_{E} \quad=\quad \sum_{p=1}^{\tau_{E}} n_{p}(p-1), \quad$ and $\tau_{E}=m_{E} N_{E}$, and $\lambda_{E_{l}}=m_{E} /\left(\Omega_{E} a_{l} \rho\right)$.

The PDF of $\gamma_{E, l}^{g, Y}$ can be easily obtained as [48]

$$
f_{\gamma_{E, l}^{g, Y}}(\gamma)=\frac{\lambda_{E_{l}}{ }^{Q \tau_{E}}}{\Gamma\left(Q \tau_{E}\right)} \gamma^{Q \tau_{E}-1} e^{-\lambda_{E l} \gamma}
$$

${ }^{1}$ Since the capabilities of the eavesdroppers are often unknown, it is reasonable to presume that they can perform multiuser decoding (e.g., successive interference cancellation). Similar assumptions were utilized in [41], [42], [45], and [46]. Definitely, the assumption is pessimistic because the eavesdropper's capability is overestimated. As a consequence the results in this work would be a lower bound of actual cases. 


\section{Secrecy Performance Analysis}

In this work, we assume that the base station has perfect CSI of all the channels. In these scenarios, selecting the best antenna aims at achieving the optimal secrecy performance [49], [50], [51]. To improve the secrecy performance, a new TAS scheme called MM is proposed, in which both users in the user pair are considered simultaneously.

The instantaneous secrecy capacity (SC) of MIMO-NOMA system can be expressed as

$$
C_{s}^{\mathrm{MM}}=\max _{g \in N_{S}}\left(\min \left(C_{i}^{g}, C_{j}^{g}\right)\right),
$$

where $C_{l}^{g}=\max \left\{\log _{2}\left(1+\gamma_{D_{l}}^{g}\right)-\log _{2}\left(1+\gamma_{E_{l}}^{g}\right), 0\right\}$ $(l \in\{i, j\})$ is the instantaneous SC of $D_{l}$ when only the $g$-th antenna at $S$ is utilized to transmit signal. The SOP of MIMONOMA systems can be expressed as ${ }^{2}$

$$
\begin{aligned}
P_{\text {out }}^{\mathrm{MM}} & =\operatorname{Pr}\left\{\max _{g \in N_{S}}\left(\min \left(C_{i}^{g}, C_{j}^{g}\right)\right) \leq R_{s}\right\} \\
& =\left(\operatorname{Pr}\left\{\min \left(C_{i}^{g}, C_{j}^{g}\right) \leq R_{s}\right\}\right)^{N_{S}} \\
& =\left(1-\operatorname{Pr}\left\{\min \left(C_{i}^{g}, C_{j}^{g}\right)>R_{s}\right\}\right)^{N_{S}} \\
& =\left(1-\operatorname{Pr}\left\{C_{i}^{g}>R_{s}\right\} \operatorname{Pr}\left\{C_{j}^{g}>R_{s}\right\}\right)^{N_{S}} \\
& =\left(1-\left(1-P_{\text {out }, i}^{g}\right)\left(1-P_{\text {out }, j}^{g}\right)\right)^{N_{S}},
\end{aligned}
$$

where $P_{\text {out }, i}^{g}$ and $P_{\text {out }, j}^{g}$ signify the SOP of $D_{i}$ and $D_{j}$ where $S$ is equipped with the $g$-th antenna (a single antenna) and $R_{s}$ denotes the target rate.

The instantaneous SC from the $g$-th antenna is expressed as [23]

$$
C_{l}^{g}=\left[\log _{2}\left(1+\gamma_{D_{l}}^{g}\right)-\log _{2}\left(1+\gamma_{E_{l}}^{g}\right)\right]^{+},
$$

where $[x]^{+}=\max \{x, 0\}$. Then we have the SOP from the $g$-th antenna

$$
\begin{aligned}
P_{\text {out }, l}^{g} & =\operatorname{Pr}\left\{C_{l}^{g} \leq R_{s}\right\} \\
& =\int_{0}^{\infty} F_{\gamma_{D_{l}}^{g}}(\Theta \gamma+\Theta-1) f_{\gamma_{E_{l}}^{g}}(\gamma) d \gamma
\end{aligned}
$$

where $\Theta=2^{R_{s}} \geq 1$.

\section{A. Eavesdroppers without collusion}

Substituting (4) and (8) into (13), and employing (3.381.4) in [52], we have

$$
\begin{aligned}
P_{o u t, j}^{g, \mathrm{~N}} & =\frac{Q \lambda_{E_{j}}^{\tau_{E}}}{\Gamma\left(\tau_{E}\right)} \sum_{k=0}^{M-j} \sum_{\Phi_{j}} \sum_{\Phi_{E}} \sum_{p=0}^{C_{D}} A_{E_{j}} \psi \\
& \times \frac{\Gamma\left(C_{E}+\tau_{E}+p\right)}{\left(B_{E_{j}}+\lambda_{E_{j}}+\frac{B_{D_{j}} \Theta}{a_{j} \rho_{D}}\right)^{C_{E}+\tau_{E}+p}} e^{-\frac{B_{D_{j}}(\Theta-1)}{a_{j} \rho}} .
\end{aligned}
$$

where $\psi=\frac{A_{D_{j}} C_{D} !(\Theta-1)^{C_{D}-p} \Theta^{p}}{p !\left(C_{D}-p\right) !\left(a_{j} \rho\right)^{C_{D}}}$.

\footnotetext{
${ }^{2}$ Note it is assumed that $C_{i}^{g}$ is independent on $C_{j}^{g}$ and vice versa, which was widely-adopted in some important works [38], [40], [41], [42], and [44]. The case that consider $C_{i}^{g}$ and $C_{j}^{g}$ dependence will be investigated in our future work based on the results in this paper.
}

Making use of (13), the SOP at $D_{i}$ can be rewritten as

$$
\begin{aligned}
P_{\text {out }, i}^{g, \mathrm{~N}} & =\underbrace{\int_{0}^{\varpi} F_{\gamma_{D_{i}}^{g}}(\Theta+\Theta \gamma-1) f_{\gamma_{E_{i}}^{g}}(\gamma) d \gamma}_{I_{1}} \\
& +\underbrace{\int_{\varpi}^{\infty} f_{\gamma_{E_{i}}^{g}}(\gamma) d \gamma}_{I_{2}},
\end{aligned}
$$

where $\varpi=\frac{1}{a_{j} \Theta}-1 \leq \frac{a_{i}}{a_{j}}$.

Utilizing (3), (8), (15), (25.4.38) of [53], we have

$$
\begin{aligned}
I_{1}^{\mathrm{N}} & =\frac{Q \lambda_{E_{i}}^{\tau_{E}}}{\Gamma\left(\tau_{E}\right)} \sum_{k=0}^{M-i} \sum_{\Phi_{i}} \sum_{\Phi_{E}} \sum_{h=1}^{H} \frac{\pi \varpi A_{D_{i}} A_{E_{i}} \sqrt{1+\varsigma_{h}^{2}}}{2 H} \\
& \times\left(\frac{\Theta+\Theta \vartheta_{h}-1}{\rho\left(a_{i}-a_{j}\left(\Theta+\Theta \vartheta_{h}-1\right)\right)}\right)^{C_{m}} \\
& \times \vartheta_{h} C_{E}+\tau_{E}-1 e^{-\frac{B_{D_{i}}\left(\Theta+\Theta \vartheta_{h}-1\right)}{\rho\left(a_{i}-a_{j}\left(\Theta+\Theta \vartheta_{h}-1\right)\right)}-\left(B_{E_{i}}+\lambda_{E_{i}}\right) \vartheta_{h}},
\end{aligned}
$$

where $H$ is the number of terms, $\varsigma_{h}=\cos \left(\frac{(2 h-1) \pi}{2 H}\right)$, and $\vartheta_{h}=\frac{\varpi\left(\varsigma_{h}+1\right)}{2}$.

Using (3.351.1) of [52], we obtain

$$
\begin{aligned}
I_{2}^{\mathrm{N}} & =1-\frac{Q \lambda_{E_{i}}^{\tau_{E}}}{\Gamma\left(\tau_{E}\right)} \sum_{\Phi_{E}} \frac{A_{E_{i}}}{\left(B_{E_{i}}+\lambda_{E_{i}}\right)^{C_{E}+\tau_{E}}} \\
& \times \Upsilon\left(C_{E}+\tau_{E},\left(B_{E_{i}}+\lambda_{E_{i}}\right) \varpi\right),
\end{aligned}
$$

where $\Upsilon(\alpha, x)=\int_{0}^{x} e^{-t} t^{\alpha-1} d t$ is the lower incomplete Gamma function, as defined by (8.350.1) in [52].

So we can obtain $P_{\text {out }, i}^{g, \mathrm{~N}}$ by substituting (16) and (17) into (15). Finally, the SOP of the MIMO-NOMA system for this case that eavesdroppers wiretap independently is obtained by substituting (14) and (15) into (11).

\section{B. Eavesdroppers with collusion}

Putting (4) and (9) into (13), and utilizing (3.351.3) of [52], we obtain the closed-form of $D_{j}$ as

$$
\begin{aligned}
P_{\text {out }, j}^{g, \mathrm{Y}}= & \frac{\lambda_{E_{j}}{ }^{Q \tau_{E}}}{\Gamma\left(Q \tau_{E}\right)} \sum_{k=0}^{M-j} \sum_{\Phi_{j}} \sum_{p=0}^{C_{D}} \psi \\
& \times \frac{\left(Q \tau_{E}+p-1\right) !}{\left(\frac{B_{D_{j}} \Theta}{a_{j} \rho}+\lambda_{E_{j}}\right)^{Q \tau_{E}+p}} e^{-\frac{B_{D_{j}}(\Theta-1)}{a_{j} \rho}} .
\end{aligned}
$$

Using (3), (9), (15), and (25.4.38) of [53], we have

$$
\begin{aligned}
& I_{1}^{\mathrm{Y}}=\frac{\lambda_{E_{i}}^{Q \tau_{E}}}{\Gamma\left(Q \tau_{E}\right)} \sum_{k=0}^{M-i} \sum_{\Phi_{i}} \sum_{h=1}^{H} e^{-\frac{B_{D_{i}}\left(\Theta+\Theta \vartheta_{h}-1\right)}{\rho\left(a_{i}-a_{j}\left(\Theta+\Theta \vartheta_{h}-1\right)\right)}-\lambda_{E_{i}} \vartheta_{h}} \\
& \times \frac{\pi \varpi A_{D_{i}} \vartheta_{h}{ }^{Q \tau_{E}-1} \sqrt{1-\varsigma_{h}^{2}}}{2 H\left(\frac{\left(\Theta+\Theta \vartheta_{h}-1\right)}{\rho\left(a_{i}-a_{j}\left(\Theta+\Theta \vartheta_{h}-1\right)\right)}\right)^{C_{D}}} .
\end{aligned}
$$

Using (3.351.1) of [52], we have

$$
I_{2}^{\mathrm{Y}}=1-\frac{\Upsilon\left(Q \tau_{E}, \lambda_{E_{i}} \varpi\right)}{\Gamma\left(Q \tau_{E}\right)} .
$$

Obviously, $P_{o u t, i}^{g, Y}$ is obtained based on (15), (19), and (20). Then the SOP of the MIMO-NOMA system with MM TAS is achieved by substituting (18) and (15) into (11). 


\section{Asymptotic Secrecy Outage Probability ANALYSIS}

To get more insights, we study the asymptotic SOP in this section. The SDO for both non-colluding and colluding eavesdroppers cases are derived when the average SNR of the main channel tends to infinity.

Based on (38) and $e^{x}=\sum_{m=0}^{k} \frac{x^{m}}{m !}+\mathcal{O}\left(x^{k}\right), F_{\tilde{H}_{S_{g} D_{j}}}^{\infty}(x)$ can be written as

$$
F_{\tilde{H}_{S_{g} D_{j}}}^{\infty}(x)=\frac{\lambda_{D}^{\tau_{D}} x^{\tau_{D}}}{\left(\tau_{D}\right) !}+\mathcal{O}\left(x^{\tau_{D}}\right)
$$

where $\lambda_{D}=m_{D} / \Omega_{D}$.

Then the asymptotic CDF of the ordered $H_{S_{g} D_{j}}$ is derived as

$$
F_{H_{S_{g} D_{j}}}^{\infty}(x)=\frac{M ! \lambda_{D}^{\tau_{D} j} x^{\tau_{D} j}}{(M-j) !(j-1) ! j\left(\left(\tau_{D}\right) !\right)^{j}} .
$$

Therefore, we obtain

$$
F_{\gamma_{D_{j}}^{g}}^{\infty}(\gamma)=\Delta_{j} \gamma^{\tau_{D} j}
$$

where $\Delta_{j}=\frac{M !\left(a_{j} \rho\right)^{-\tau_{D} D^{j}} \lambda_{D}^{\tau_{D}^{j}}}{(M-j) !(j-1) ! j\left(\left(\tau_{D}\right) !\right)^{j}}$.

Similarly, we have

$$
F_{\gamma_{D_{i}}^{g}}^{\infty}(\gamma)=\frac{\Delta_{i} \gamma^{\tau_{D} i}}{\left(a_{i}-a_{j} \gamma\right)^{\tau_{D} i}},
$$

where $\Delta_{i}=\frac{M ! \rho^{-\tau_{D^{i}}} \lambda_{D}^{\tau_{D}{ }^{i}}}{(M-i) !(i-1) ! i\left(\left(\tau_{D}\right) !\right)^{i}}$.

Substituting (8), (9) and (23) into (13), and using (3.351.3) of [52], the asymptotic SOP at $D_{j}$ can be rewritten as

$$
\begin{gathered}
P_{o u t, j}^{\infty, g, \mathrm{~N}}=\frac{\Delta_{j} Q \lambda_{E_{j}}^{\tau_{E}}}{\Gamma\left(\tau_{E}\right)} \sum_{\Phi_{E}} \sum_{p=0}^{\tau_{D} j} \frac{\phi A_{E_{j}} \Gamma\left(C_{E}+\tau_{E}+p\right)}{\left(B_{E_{j}}+\lambda_{E_{j}}\right)^{C_{E}+\tau_{E}+p}}, \\
P_{\text {out }, j}^{\infty, g, \mathrm{Y}}=\frac{\Delta_{j} \lambda_{E_{j}}^{Q \tau_{E}}}{\Gamma\left(Q \tau_{E}\right)} \sum_{p=0}^{\tau_{D} j} \frac{\phi \Gamma\left(Q \tau_{E}+p\right)}{\lambda_{E_{j}}{ }^{Q \tau_{E}+p}},
\end{gathered}
$$

respectively, where $\phi=\frac{\left(\tau_{D} j\right) !\left(\Theta-1 \tau^{\tau^{j}-p} \Theta^{p}\right.}{p !\left(\tau_{D} j-p\right) !}$.

Making use of (8), (9), (15), (24), and (25.4.38) of [53], we obtain

$$
\begin{aligned}
I_{1}^{\infty, \mathrm{N}}= & \frac{\Delta_{i} Q \lambda_{E_{i}}^{\tau_{E}}}{\Gamma\left(\tau_{E}\right)} \sum_{\Phi_{E}} \sum_{h=1}^{H} \frac{\pi \varpi A_{E_{i}} \sqrt{1-\varsigma_{h}}}{2 H} \\
\times & \frac{\left(\Theta+\Theta \vartheta_{h}-1\right)^{\tau_{D} i} \vartheta_{h} C_{E}+\tau_{E}-1}{\left(a_{i}-a_{j}\left(\Theta+\Theta \vartheta_{h}-1\right)\right)^{\tau_{D} i}} e^{-\left(B_{E_{i}}+\lambda_{E_{i}}\right) \vartheta_{h}} \\
I_{1}^{\infty, \mathrm{Y}} & =\frac{\Delta_{i} \lambda_{E_{i}}^{Q \tau_{E}}}{\Gamma\left(Q \tau_{E}\right)} \sum_{h=1}^{H} \frac{\pi \varpi \sqrt{1-\varsigma_{h}^{2}}}{2 H} \\
& \times \frac{\left(\Theta+\Theta \vartheta_{h}-1\right)^{\tau_{D} i} \vartheta_{h}{ }^{Q \tau_{E}-1}}{\left(a_{i}-a_{j}\left(\Theta+\Theta \vartheta_{h}-1\right)\right)^{\tau_{D} i}} e^{-\lambda_{E_{i}} \vartheta_{h}}
\end{aligned}
$$

respectively.

Note we have $I_{2}^{\infty, \mathrm{N}}=I_{2}^{\mathrm{N}}$ and $I_{2}^{\infty, \mathrm{Y}}=I_{2}^{\mathrm{Y}}$ since $I_{2}$ only depend on the PDF of $\gamma_{E}$. Then the SOP for MIMO-NOMA systems with MM TAS can be achieved by substituting $P_{\text {out }, i}^{\infty, g}$ and $P_{o u t, j}^{\infty, g}$ into (11).
The SDO can be expressed as [50]

$$
G_{d, l}=-\lim _{\Omega_{D} \rightarrow \infty} \frac{\ln P_{\text {out }, l}^{\infty}}{\ln \Omega_{D}},
$$

where $P_{\text {out }, l}^{\infty}$ denotes the asymptotic SOP. Then SDO in both case can be easily obtained as

$$
G_{d}^{\mathrm{MM}, 1}=N_{S}\left(\min \left\{G_{d, i}, G_{d, j}\right\}\right)=0 .
$$

Remark 1: Based on (15), (17), (20), (25)-(29), one can easily achieve the SDO of the $j$-th and $i$-th receivers in MIMONOMA systems with fixed PA in both cases are $\tau_{D} j$ and 0 , respectively. That is to say, multiple antennas only improve the SDO of the near user but has no influence on the far user. Furthermore, whether collusion between eavesdroppers or not does not affect the SDO of each user.

This phenomenon is comprehensible. The SOP of user $i$ was given by (15), which equates the sum of $I_{1}$ and $I_{2}$. Obviously, increasing main channel gains $\left(\Omega_{D}\right)$ will decrease $I_{1}$ but has no influence on $I_{2}$. That is to say, $I_{2}$ will be the dominant part of $P_{\text {out }, i}$ and is constant when $\Omega_{D} \rightarrow \infty$. From another point of view, using (1), we can achieve that while $\Omega_{D}$ increasing, the received SNR at $i$ has an upper bound, which is a constant $\frac{a_{i}}{a_{j}}$. Namely, we have $\gamma_{D_{i}}^{g} \stackrel{\Omega_{D} \rightarrow \infty}{\rightarrow} \frac{a_{i}}{a_{j}}$. Thus $P_{\text {out }, i}$ will be a constant when $\Omega_{D} \rightarrow \infty$. Further, the SDO of user $i$ is zero.

\section{Secrecy Outage Probability with Dynamic Power Allocation Method}

The results in Section IV show that the SDO of NOMA systems is determined by the far user (user $i$ in this work). To improve the SDO of the user $i, I_{2}$ must decrease with increasing $\Omega_{D}$. Fortunately, it can be realized by adjusting $\varpi$, which has an upper bound as $\frac{a_{i}}{a_{i}}$. A new dynamic PA scheme has been proposed in [38] and the results there verified that it can obtain non-zeros SDO. The main idea of this dynamic PA scheme is to let $\frac{a_{i}}{a_{j}}$ increase with the increasing of $\Omega_{D}$. Thereby $I_{2}$ will decrease since the integration interval diminishes. In this section, we analyze the SOP and SDO of MIMO-NOMA systems when this dynamic PA scheme is utilized on all the legitimate users.

Let $\frac{a_{i}}{a_{j}}=\alpha \lambda_{D}^{-\omega}(0<\omega<1, \alpha>1)^{3}$. As $a_{i}+a_{j}=1$, we obtain

$$
\left\{\begin{array}{l}
a_{j}=\frac{1}{1+\alpha \lambda_{D}-\omega} \\
a_{i}=\frac{\alpha \lambda_{D}-\omega}{1+\alpha \lambda_{D}-\omega}
\end{array} .\right.
$$

Using (24), (31), and $e^{x}=\sum_{m=0}^{k} \frac{x^{m}}{m !}+\mathcal{O}\left(x^{k}\right)$, we obtain $F_{\gamma_{D_{2}}}^{\infty}(\gamma)$ as

$$
F_{\gamma_{D_{i}}^{g}}^{\infty, \text { non }}(\gamma)=\frac{\Delta_{i} \gamma^{\tau_{D} i}}{a_{i} \tau_{D} i}
$$

\footnotetext{
${ }^{3} \alpha>1$ is to obtain the fairness and make sure that user $i$ has more power than user $j\left(a_{i}>a_{j}\right)$ and $0<\omega<1$ is to make sure that the SNR and SINR at user $i$ and $j$ increases with $\Omega_{D}$ simultaneously.
} 
Substituting (31) into (25) and (26), we obtain the asymptotic SOP at $D_{1}$ as

$$
\begin{aligned}
P_{\text {out }, j}^{\infty, g, \text { non }, \mathrm{N}} & =\frac{\Delta_{j}^{\text {non }}\left(1+\alpha \lambda_{D}{ }^{-\omega}\right)^{\tau_{D} j} \lambda_{D}{ }^{\tau_{D} j} Q \lambda_{E_{j}}{ }^{\tau_{E}}}{\Gamma\left(\tau_{E}\right)} \\
& \times \sum_{\Phi_{E}} \sum_{p=0}^{\tau_{D} j} \frac{\phi A_{E_{j}} \Gamma\left(C_{E}+\tau_{E}+p\right)}{\left(B_{E_{j}}+\lambda_{E_{j}}\right)^{C_{E}+\tau_{E}+p}}, \\
P_{\text {out }, j}^{\infty, g, \text { non }, \mathrm{Y}} & =\frac{\Delta_{j}^{\text {non }}\left(1+\alpha \lambda_{D}{ }^{-\omega}\right)^{\tau_{D} j} \lambda_{D}{ }^{\tau_{D} j} \lambda_{E_{j}}{ }^{Q \tau_{E}}}{\Gamma\left(Q \tau_{E}\right)} \\
& \times \sum_{p=0}^{\tau_{D} j} \frac{\phi \Gamma\left(Q \tau_{E}+p\right)}{\lambda_{E_{j}}{ }^{Q \tau_{E}+p}},
\end{aligned}
$$

respectively, where $\Delta_{j}^{\text {non }}=\frac{M ! \rho^{-\tau_{D}}}{(M-j) !(j-1) ! j\left(\left(\tau_{D}\right) !\right)^{j}}$.

After putting (6), (7) and (32) into (13) and employing (3.381.4) of [52], we get

$$
\begin{aligned}
P_{\text {out }, i}^{\infty, g, \text { non }, \mathrm{N}} & =\sum_{\Phi_{E}} \sum_{p=0}^{\tau_{D} i} \frac{\Delta_{i}^{\text {non }} Q \lambda_{E_{i}}{ }^{\tau_{E}} A_{E_{i}} \lambda_{D}{ }^{\tau_{D} i}\left(1+\alpha \lambda_{D}{ }^{-\omega}\right)^{\tau_{D} i}}{\Gamma\left(\tau_{E}\right) \alpha \lambda_{D}{ }^{-\omega \tau_{D} i}} \\
& \times \frac{\Upsilon\left(C_{E}+\tau_{E}+p,\left(B_{E_{i}}+\lambda_{E_{i}}\right) \varpi\right)}{\left(B_{E_{i}}+\lambda_{E_{i}}\right)^{C_{E}+\tau_{E}+p}} \\
P_{\text {out }, i}^{\infty, g, \text { non }, \mathrm{Y}} & =\sum_{p=0}^{\tau_{D} i} \frac{\Delta_{i}^{\text {non }} \lambda_{E_{i}}{ }^{Q \tau_{E}} \lambda_{D}{ }^{\tau_{D} i}\left(1+\alpha \lambda_{D}{ }^{-\omega}\right)^{\tau_{D} i}}{\Gamma\left(Q \tau_{E}\right) \alpha \lambda_{D}{ }^{-\omega \tau_{D} i}} \\
& \times \frac{\Upsilon\left(Q \tau_{E}+p, \lambda_{E_{i}} \varpi\right)}{\lambda_{E_{i}}{ }^{C_{E}+\tau_{E}+p}}
\end{aligned}
$$

respectively, where $\Delta_{i}^{\text {non }}=\frac{M ! \rho^{-\tau_{D}}\left(\tau_{D} i\right) !(\Theta-1)^{\tau} D^{i-p} \Theta^{p}}{(M-i) !(i-1) ! i\left(\left(\tau_{D}\right) !\right)^{i} p !\left(\tau_{D} i-p\right) !}$.

Based on (33) - (36), the SDO of user $i$ and $j$ with the dynamic PA scheme is obtained as $\tau_{D} i$ and $(1-\omega) \tau_{D} j$, respectively. Then we get the SDO of the MIMO-NOMA systems with dynamic PA is improved as

$$
G_{d}^{\mathrm{MM}, 2}=N_{S} \min \left\{\tau_{D} i,(1-\omega) \tau_{D} j\right\} .
$$

Remark 2: From (37), $\omega$ exhibits a strong influence on the SDO of user $j$, which means enhancing the SDO of user $i$ is at the cost of reducing the SDO of user $j$. Furthermore, the SDO for MIMO-NOMA systems with dynamic PA will reduce as $\omega$ increases and there is a ceiling as $N_{S} \tau_{D} i$, which is proved by Fig. 8 in this work.

Remark 3: Another interesting phenomenon can be observed from (37), i.e., the SDO of each user not only depend on the number of antennas at base station, the number of antennas at himself, and the fading parameter of the main channels, but also depend on their ordered index. Moreover, the SDO is independent of the number of eavesdropper, the number of antennas at each eavesdropper, and the fading parameter of the wiretap channels.

\section{NUMERICAL RESULTS}

Monte-Carlo simulation is given to corroborate our results. The main adopted parameters are set as $R_{s}=0.1 \mathrm{bit} / \mathrm{s} / \mathrm{Hz}$, $\sigma^{2}=1, m_{D}=m_{E}=2$, and $P_{S}=0 \mathrm{~dB}$. The PA scheme in Figs. 2-5 is fixed and in Figs. 6-8 is dynamic. One can observe that the analysis results match perfectly with simulation.

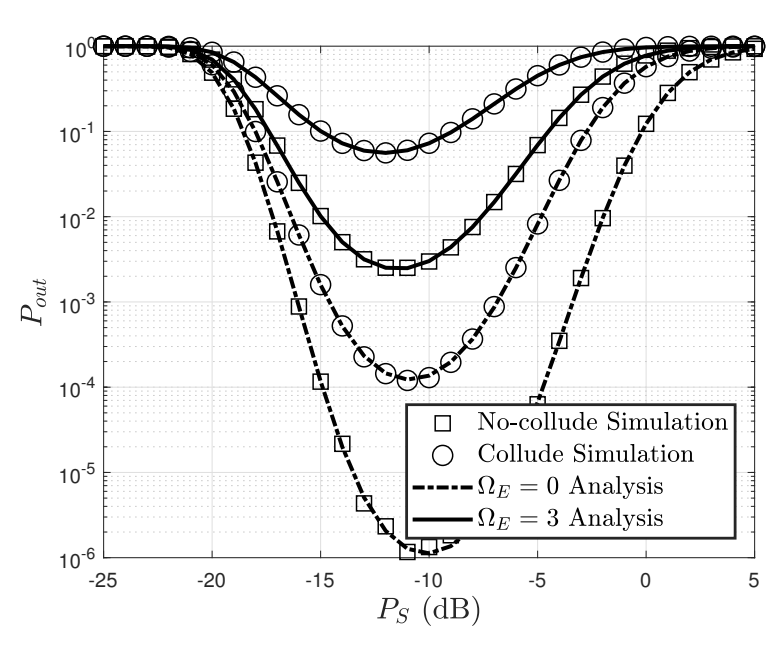

Fig. 2. SOP for various $P_{S}$ with $\Omega_{D}=10 \mathrm{~dB}, N_{D}=N_{E}=N_{S}=3$, $Q=2, M=4, i=1, j=4, a_{j}=0.2$, and $a_{i}=0.8$,

It is showed in Fig. 2 that the SOP is improved while the transmit power increasing. Then it degrades in the high $P_{S}$ region before becomes a constant, which is similar to the results in [38]. We can also observe that the SOP with eavesdropper-collusion is inferior compared to the case that without collusion, this is because collusion can enhance the performance of the eavesdroppers. Furthermore, one can find that the SOP for a lower $\Omega_{E}$ are better than the ones for a higher $\Omega_{E}$ since larger $\Omega_{E}$ means better channel conditions at the eavesdropper. From Fig. 2, we can draw an interesting conclusion that there is an optimal transmit power that can obtain the best secrecy performance for MIMO-NOMA system, similar to [38].

It is verified in Fig. 3 that the SOP is enhanced by increasing $\Omega_{D}$, since the increased $\Omega_{D}$ means that the channel condition of the legitimate destination is improved. Based on (15), we know that the SOP equals to the summation of $I_{1}$ and $I_{2}$. When $\Omega_{D}$ increases, $I_{2}$ will be dominant. Therefore, the SOP will reach a floor when $\Omega_{D}$ tends to infinity because there exists a ceiling for the secrecy capacity, which is testified in [54]. Moreover, the security outage performance is improved as the number of antennas at the destination increases, since the SNR at the destination will be increased.

Fig. 4 presents the SOP versus various number of antennas at $S$. We can see that the SOP is enhanced as $N_{S}$ increases or $N_{E}$ decreases, this is because the transmitting diversity of legitimate users will be enhanced while increasing $N_{S}$ and the receiving diversity of eavesdroppers will become weak with decreasing $N_{E}$.

Fig. 5 presents the SOP for different legitimate users with fixed PA. One can observe that the SOP increases when $i$ increases. But the change of $j$ almost has no influence on the SOP, which means SOP of the MIMO-NOMA system mainly depends on SOP of the $i$-th user, which has lower channel gains. The secrecy performance of user $i$ should be considered emphatically if we want to enrich the security of the whole NOMA systems.

The SOP with dynamic PA scheme is presented in Fig. 6. it 


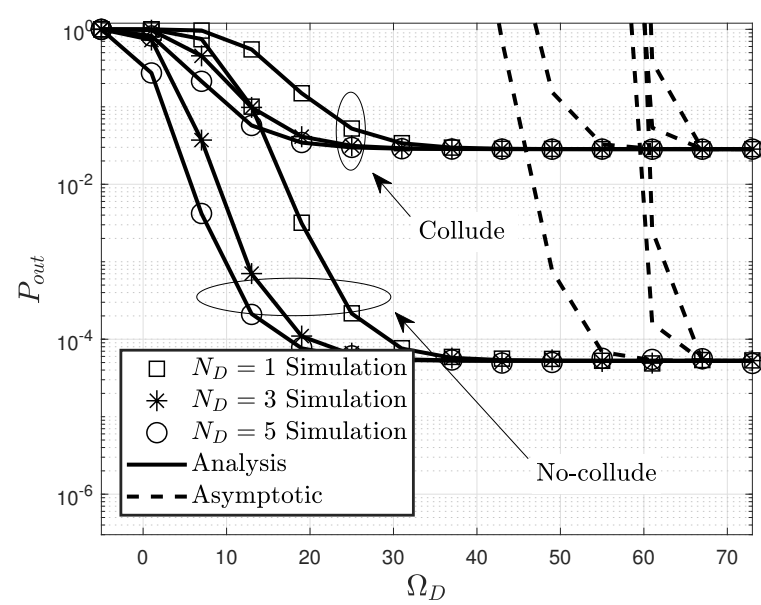

Fig. 3. SOP for various $\Omega_{D}$ with $\Omega_{E}=0 \mathrm{~dB}, N_{S}=3, N_{E}=2, Q=2$, $M=4, i=1, j=4, a_{j}=0.2$, and $a_{i}=0.8$.

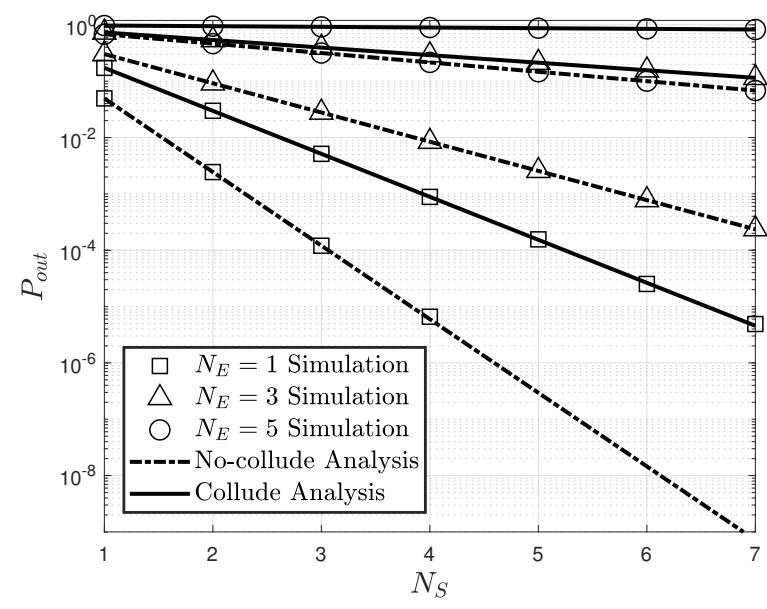

Fig. 4. SOP for various $N_{S}$ with $\Omega_{D}=10 \mathrm{~dB}, \Omega_{E}=0 \mathrm{~dB}, N_{D}=Q=2$, $M=4, i=1, j=3, a_{j}=0.2$, and $a_{i}=0.8$.

can be found that the non-zero SDO is achieved, as confirmed by (37). In addition, the SOP will degrade as increasing the number of eavesdroppers $(Q)$, which is equivalent to enhance the quality of the eavesdropping channel.

In Fig. 7, we compare the SOP for different $m$ versus $\Omega_{D}$. One can find that the SOP with larger $m$ outperforms the one with a small $m$ because a larger $m$ implies that the channel fading is weak.

Fig. 8 presents the SOP for different $\omega$ when there is collusion among the eavesdroppers. We can observe that decreasing $\omega$ will improve the SDO of systems with dynamic PA, which is stated in Remark 2. There is a maximal SDO, which is equal to $N_{S} \tau_{D} i$ and does not depend on $\omega$.

\section{CONCLUSION}

In this work, we proposed the MM TAS scheme to improve the SOP of a MIMO-NOMA system. The exact and asymptotic closed-form expressions for SOP are derived when non-colluding and colluding schemes are utilized for the

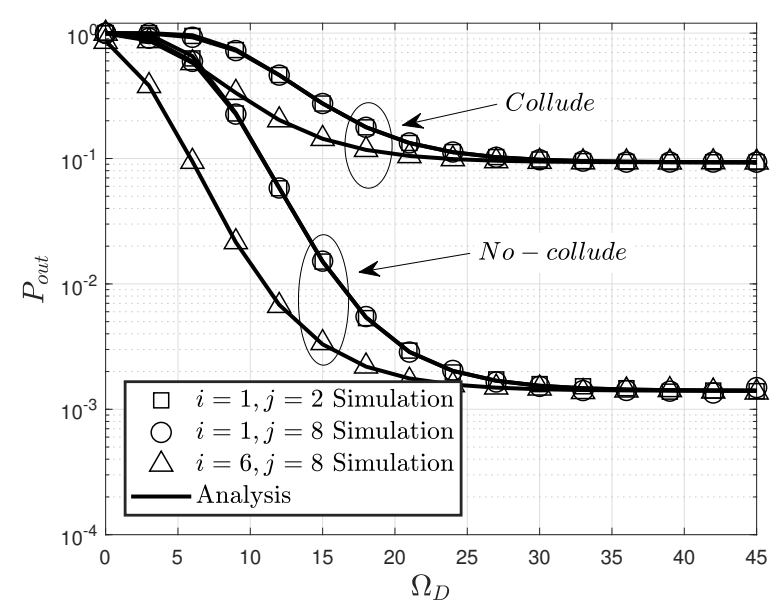

Fig. 5. SOP for various $\Omega_{D}$ with $\Omega_{E}=0 \mathrm{~dB}, N_{S}=N_{D}=N_{E}=2$, $Q=2, M=4, a_{j}=0.2$, and $a_{i}=0.8$.

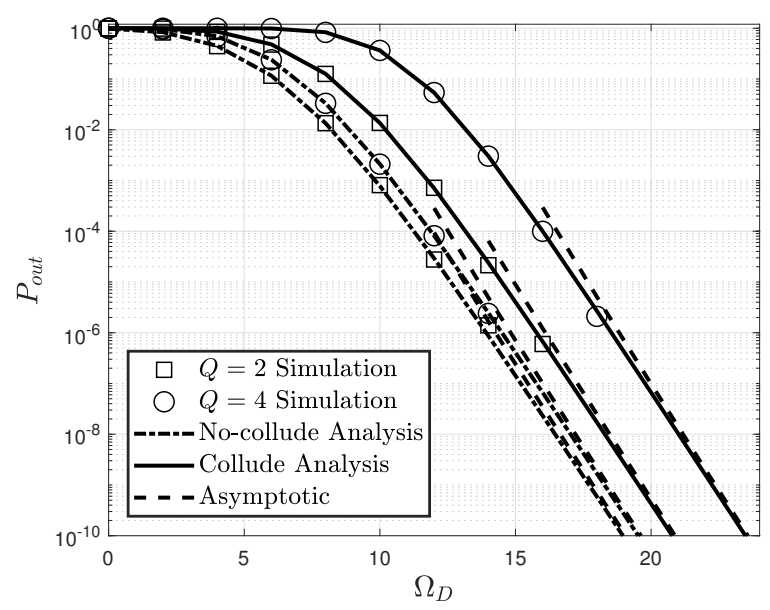

Fig. 6. SOP with dynamic PA scheme for various $\Omega_{D}$ with $\Omega_{E}=0 \mathrm{~dB}$, $N_{S}=N_{E}=N_{D}=2, M=4, i=1, j=4, \alpha=11$, and $\omega=0.6$.

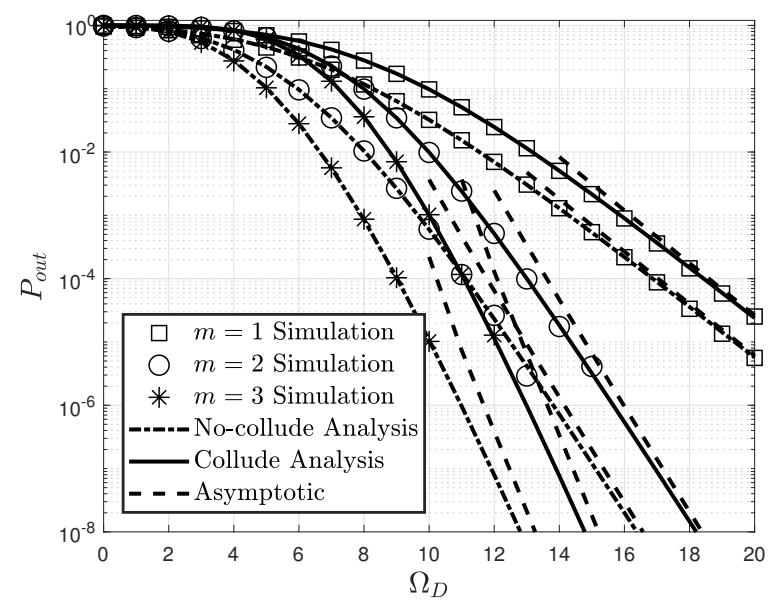

Fig. 7. SOP with dynamic PA scheme for various $\Omega_{D}$ with $\Omega_{E}=0 \mathrm{~dB}$, $N_{S}=N_{E}=N_{D}=2, Q=2, M=4, i=1, j=4, \alpha=10$, and $\omega=0.6$. 


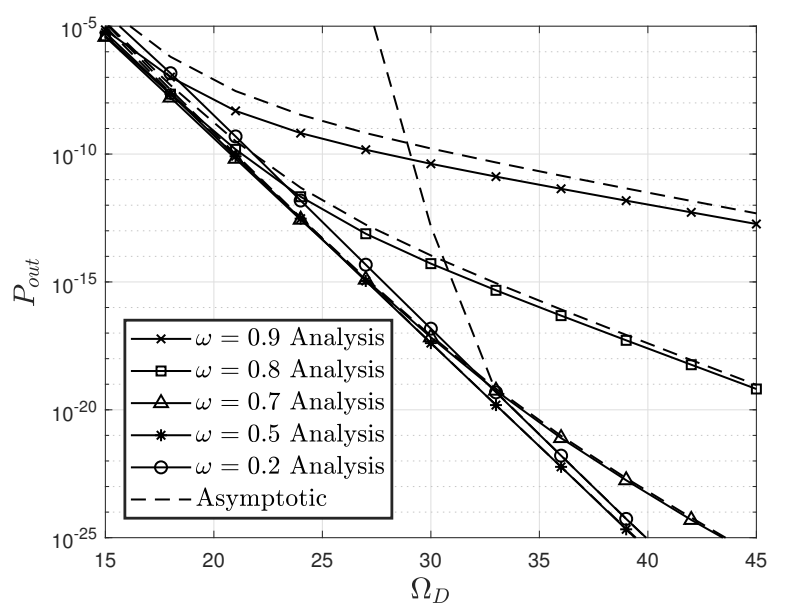

Fig. 8. SOP with dynamic PA scheme for various $\Omega_{D}$ with $\Omega_{E}=0 \mathrm{~dB}$, $N_{S}=N_{E}=N_{D}=2, Q=2, M=4, i=1, j=2$, and $\alpha=10$ when there is a collusion among the eavesdroppers.

eavesdroppers. Monte-Carlo simulation results were given to verify our analytical results. The results show that when the MM TAS scheme is adopted, increasing the number of the antennas at base station can significantly improve the SOP of the MIMO-NOMA system. Further, the ordered index has an important influence on the SDO of each user. Although it was found that there is an optimal transmit power that can obtain the minimum secrecy outage, how to obtain the closedform expression of the optimal transmit power would be an interesting task. Moreover, there are some other important research issues that could be done in future work based on the results of this work, such as cooperative NOMA systems, cognitive NOMA systems and NOMA systems with energy harvesting. Another interesting future topic is to analyze the impact of imperfect CSI on energy efficiency of secure NOMA systems, whereas the energy-efficient optimization problem for NOMA systems with imperfect CSI were solved in [56].

\section{APPENDIX A}

The CDF of the unordered power gains $\tilde{H}_{S_{g} D_{j}}=$ $\sum_{k=1}^{N_{D}}\left|h_{S_{g} D_{j, k}}\right|^{2}$ is obtained as [48]

$$
F_{\tilde{H}_{S_{g} D_{j}}}(x)=1-e^{\left(-\frac{m_{D} x}{\Omega_{D}}\right)} \sum_{k=0}^{\tau_{D}-1} \frac{1}{k !}\left(\frac{m_{D} x}{\Omega_{D}}\right)^{k}
$$

where $\Omega_{D}$ means the average SNR.

Based on order statistics [55] and multinomial theorem [47], the CDF of the ordered power gains $H_{S_{g} D_{j}}$ is obtained as

$$
\begin{aligned}
F_{H_{S_{g} D_{j}}}(x)= & \frac{M !}{(j-1) !} \sum_{k=0}^{M-j} \frac{(-1)^{k}}{(j+k)(M-j-k) ! k !} \\
& \times\left(F_{\tilde{H}_{S_{g} D_{j}}}(x)\right)^{j+k} \\
= & \sum_{k=0}^{M-j} \sum_{\Phi_{j}} A_{D_{j}} e^{-B_{D_{j}} x} x^{C_{D}},
\end{aligned}
$$

where $\Phi_{j}=\left\{\left(n_{1}, \cdots, n_{\tau_{D}+1}\right) \in \mathbb{Z} \geq \mid \sum_{p=1}^{\tau_{D}+1} n_{p}=j+k\right\}$,

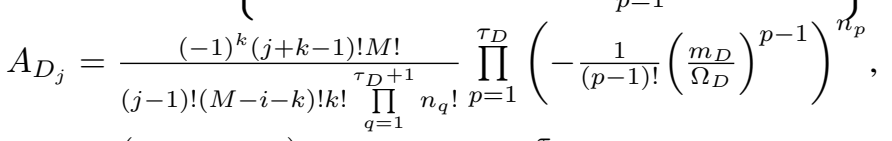
$B_{D_{j}}=\frac{\left(j+k-n_{\tau_{D}+1}\right) m_{D}}{\Omega_{D}}$, and $C_{D}=\sum_{p=1}^{\tau_{D}} n_{p}(p-1)$.

Making use of (2) and (39), we obtain

$$
F_{\gamma_{D_{j}}^{g}}(\gamma)=\sum_{k=0}^{M-j} \sum_{\Phi_{j}} A_{D_{j}}\left(a_{j} \rho_{D}\right)^{-C_{D}} e^{-\frac{B_{D_{j}}}{a_{j} \rho_{D}} \gamma} \gamma^{C_{D}}
$$

Similarly, $F_{\gamma_{D_{i}}^{g}}(\gamma)$ is obtained as

$$
F_{\gamma_{D_{i}}^{g}}(\gamma)=\left\{\begin{array}{lc}
\sum_{k=0}^{M-i} \sum_{\Phi_{i}} A_{D_{i}} e^{-B_{D_{i}} \varphi(\gamma)}(\varphi(\gamma))^{C_{D}}, & \gamma<\frac{a_{i}}{a_{j}} \\
1, & \gamma \geq \frac{a_{i}}{a_{j}}
\end{array}\right.
$$

where $\Phi_{i}=\left\{\left(n_{1}, \cdots, n_{\tau_{D}+1}\right) \in \mathbb{Z}^{\geq} \mid \sum_{p=1}^{\tau_{D}+1} n_{p}=i+k\right\}$, $A_{D_{i}}=\frac{(-1)^{k}(i+k-1) ! M !}{(i-1) !(M-i-k) ! k ! \prod_{q=1}^{\tau_{D}+1} n_{q} !} \prod_{p=1}^{\tau_{D}}\left(-\frac{1}{(p-1) !}\left(\frac{m_{D}}{\Omega_{D}}\right)^{p-1}\right)^{n_{p}}$ $B_{D_{i}}=\frac{\left(i+k-n_{\tau_{D}+1}\right) m_{D}}{\Omega_{D}}$, and $\varphi(\gamma)=\frac{\gamma}{\rho_{D}\left(a_{i}-a_{j} \gamma\right)}$.

\section{REFERENCES}

[1] J. N. Laneman, D. N. C. Tse, and G. W. Wornell, "Cooperative diversity in wireless networks: Efficient protocols and outage behavior," IEEE Trans. Inf. Theory, vol. 50, no. 12, pp. 3062-3080, Dec. 2004.

[2] H. Zhang, C. Jiang, N. C. Beaulieu, X. Chu, X. Wang, and T. Q. S. Quek, "Resource allocation for cognitive small cell networks: A cooperative bargaining game theoretic approach," IEEE Trans. Wireless Commun., vol. 14, no. 6, pp. 3481-3493, Jun. 2015.

[3] Z. Ding, Y. Liu, J. Choi, Q. Sun, M. Elkashlan, C. -L. I, and H. V. Poor, "Application of non-orthogonal multiple access in LTE and 5G networks," IEEE Commun. Mag., vol. 55, no. 2, pp. 185-191, Feb. 2017.

[4] L. Dai, B. Wang, Y. Yuan, S. Han, C. -L. I, and Z. Wang, "Non-orthogonal multiple access for $5 \mathrm{G}$ : Solutions, challenges, opportunities, and future research trends," IEEE Commun. Mag., vol. 53, no. 9, pp. 74-81, Sep. 2015.

[5] Z. Ding, X. Lei, G. K. Karagiannidis, R. Schober, J. Yuan, and V. K. Bhargava, "A survey on non-orthogonal multiple access for $5 \mathrm{G}$ networks: Research challenges and future trends," IEEE J. Sel. Areas Commun., vol. 35, no. 10, pp. 2181-2195, Oct. 2017.

[6] P. Xu, Y. Yuan, Z. Ding, X. Dai, and R. Schober, "On the outage performance of non-orthogonal multiple access with 1-bit feedback," IEEE Trans. Wireless Commun., vol. 15, no. 10, pp. 6716-6730, Oct. 2016.

[7] P. Xu and K. Cumanan, "Optimal power allocation scheme for nonorthogonal multiple access with alpha-fairness," IEEE J. Sel. Areas Commun., vol. 35, no. 10, pp. 2357-2369, Oct. 2017.

[8] Z. Ding, Z. Yang, P. Fan, and H. V. Poor, "On the performance of nonorthogonal multiple access in $5 \mathrm{G}$ systems with randomly deployed users," IEEE Signal Process. Lett., vol. 21, no. 12, pp. 1501-1505, Dec. 2014.

[9] S. Timotheou and I. Krikidis, "Fairness for non-orthogonal multiple access in 5G systems," IEEE Signal Process. Lett., vol. 22, no. 10, pp. 1647-1651, Oct. 2015.

[10] Y. Liu, Z. Ding, M. Elkashlan, and J. Yuan, "Non-orthogonal multiple access in large-scale underlay cognitive radio networks," IEEE Trans. Veh. Technol., vol. 65, no. 12, pp. 10152-10157, Dec. 2016.

[11] J. Men, J. Ge, and C. Zhang, "Performance analysis of non-orthogonal multiple access for relaying networks over Nakagami- $m$ fading channels," IEEE Trans. Veh. Technol., vol. 66, no. 2, pp. 1200-1208, Feb. 2017.

[12] Z. Ding, F. Adachi, and H. V. Poor, "The application of MIMO to nonorthogonal multiple access," IEEE Trans. Wireless Commun., vol. 15, no. 1, pp. 537-552, Jan. 2016. 
[13] Z. Ding, R. Schober, and H. V. Poor, "A general MIMO framework for NOMA downlink and uplink transmission based on signal alignment," IEEE Trans. Wireless Commun., vol. 15, no. 6, pp. 4438-4454, Jun. 2016.

[14] X. Chen, Z. Zhang, C. Zhong, and D. W. K. Ng, "Exploiting multipleantenna techniques for non-orthogonal multiple access," IEEE J. Sel. Areas Commun., vol. 35, no. 10, pp. 2207-2220, Oct. 2017.

[15] Q. Sun, S. Han, I. C. L, and Z. Pan, "On the ergodic capacity of MIMO NOMA systems," IEEE Wireless Commun. Lett., vol. 4, no. 4, pp. 405408, Aug. 2015.

[16] Y. Liu, G. Pan, H. Zhang, and M. Song, "On the capacity comparison between MIMO-NOMA and MIMO-OMA," IEEE Access, vol. 4, pp. 2123-2129, May 2016.

[17] W. Han, J. Ge, and J. Men, "Performance analysis for NOMA energy harvesting relaying networks with transmit antenna selection and maximal-ratio combining over Nakagami-m fading," IET Commun., vol. 10, no. 18, pp. 2687-2693, Dec. 2016.

[18] N. T. Do, D. B. d. Costa, T. Q. Duong, and B. An, "Transmit antenna selection schemes for MISO-NOMA cooperative downlink transmissions with hybrid SWIPT protocol," in 2017 IEEE International Conference on Communications (ICC), Paris, May 2017, pp. 1-6.

[19] A. P. Shrestha, T. Han, Z. Bai, J. M. Kim, and K. S. Kwak, "Performance of transmit antenna selection in non-orthogonal multiple access for 5G systems," in 2016 Eighth International Conference on Ubiquitous and Future Networks (ICUFN), Vienna, Jul. 2016, pp. 1031-1034.

[20] X. Liu and X. Wang, "Efficient antenna selection and user scheduling in 5G massive MIMO-NOMA system," in 2016 IEEE 83rd Vehicular Technology Conference (VTC Spring), Nanjing, May 2016, pp. 1-5.

[21] Y. Yu, H. Chen, Y. Li, Z. Ding, and B. Vucetic, "Antenna selection for MIMO-NOMA networks," in 2017 IEEE International Conference on Communications (ICC), Paris, May 2017, pp. 1-6.

[22] Y. Yu, H. Chen, Y. Li, Z. Ding, and L. Zhuo, "Antenna selection in MIMO cognitive radio-inspired NOMA systems," IEEE Commun. Lett., vol. 21, no. 12, pp. 2658-2661, Dec 2017.

[23] M. Bloch, J. Barros, M. R. D. Rodrigues, and S. W. McLaughlin, "Wireless information-theoretic security," IEEE Trans. Inf. Theory, vol. 54 , no. 6, pp. 2515-2534, Jun. 2008

[24] S. Gong, C. Xing, S. Chen, and Z. Fei, "Secure communications for dual-polarized MIMO systems," IEEE Trans. Signal Process., vol. 65, no. 16, pp. 4177-4192, Aug. 2017.

[25] R. Bassily, E. Ekrem, X. He, E. Tekin, J. W. Xie, M. R. Bloch, S. Ulukus, and A. Yener, "Cooperative security at the physical layer: A summary of recent advances," IEEE Signal Process. Mag., vol. 30, no. 5, pp. 16-28, Sep 2013

[26] Y.-W. P. Hong, P.-C. Lan, and C.-C. J. Kuo, "Enhancing physicallayer secrecy in multiantenna wireless systems: An overview of signal processing approaches," IEEE Signal Process. Mag., vol. 30, no. 5, pp. 29-40, Sept. 2013.

[27] A. Mukherjee, S. A. A. Fakoorian, J. Huang, and A. L. Swindlehurst, "Principles of physical layer security in multiuser wireless networks: A survey," IEEE Commun. Surveys Tuts., vol. 16, no. 3, pp. 1550-1573, 3nd Quart. 2014.

[28] W. Trappe, "The challenges facing physical layer security," IEEE Commun. Mag., vol. 53, no. 6, pp. 16-20, Jun. 2015.

[29] N. Yang, L. Wang, G. Geraci, M. Elkashlan, J. Yuan, and M. D. Renzo, "Safeguarding 5G wireless communication networks using physical layer security," IEEE Commun. Mag., vol. 53, no. 4, pp. 20-27, Apr. 2015

[30] Y. Zou, J. Zhu, X. Wang, and L. Hanzo, "A survey on wireless security: Technical challenges, recent advances, and future trends," Proc. IEEE, vol. 104, no. 9, pp. 1727-1765, May 2016.

[31] H. Lei, H. Zhang, I. S. Ansari, C. Gao, Y. Guo, G. Pan, and K. A. Qaraqe, "Secrecy outage performance for SIMO underlay cognitive radio systems with generalized selection combining over Nakagami- $m$ channels," IEEE Trans. Veh. Technol., vol. 65, no. 12, pp. 10126-10132, Dec. 2016.

[32] Y. Liu, H. H. Chen, and L. Wang, "Physical layer security for next generation wireless networks: Theories, technologies, and challenges," IEEE Commun. Surveys Tuts., vol. 19, no. 1, pp. 347-376, 1st Quart. 2017.

[33] X. Chen, D. W. K. Ng, W. H. Gerstacker, and H. H. Chen, "A survey on multiple-antenna techniques for physical layer security," IEEE Commun. Surveys Tuts., vol. 19, no. 2, pp. 1027-1053, 2nd Quart. 2017.

[34] H. Zhang, H. Xing, J. Cheng, A. Nallanathan, and V. C. M. Leung, "Secure resource allocation for OFDMA two-way relay wireless sensor networks without and with cooperative jamming," IEEE Trans. Ind. Informat., vol. 12, no. 5, pp. 1714-1725, Oct. 2016.

[35] N. Zhao, F. R. Yu, M. Li, Q. Yan, and V. C. M. Leung, "Physical layer security issues in interference-alignment-based wireless networks," IEEE Commun. Mag., vol. 54, no. 8, pp. 162-168, Aug. 2016.
[36] N. Zhao, F. R. Yu, M. Li, and V. C. M. Leung, "Anti-eavesdropping schemes for interference alignment (IA)-based wireless networks," IEEE Trans. Wireless Commun., vol. 15, no. 8, pp. 5719-5732, Aug. 2016.

[37] Y. Zhang, H. M. Wang, Q. Yang, and Z. Ding, "Secrecy sum rate maximization in non-orthogonal multiple access," IEEE Commun. Lett., vol. 20, no. 5, pp. 930-933, May 2016.

[38] H. Lei, J. Zhang, K.-H. Park, P. Xu, I. S. Ansari, G. Pan, B. Alomair, and M.-S. Alouini, "On secrecy performance of NOMA system with transmit antenna selection," IEEE Access, vol. 5, no. 1, pp. 17450 - 17464, Sept. 2017.

[39] L. Lv, Q. Ni, Z. Ding, and J. Chen, "Cooperative non-orthogonal relaying for security enhancement in untrusted relay networks," in 2017 IEEE International Conference on Communications (ICC), Paris, May 2017, pp. 1-6.

[40] B. He, A. Liu, N. Yang, and V. K. N. Lau, "On the design of secure non-orthogonal multiple access systems," IEEE J. Sel. Areas Commun., vol. 35, no. 10, pp. 2196-2206, Oct. 2017.

[41] Z. Qin, Y. Liu, Z. Ding, Y. Gao, and M. Elkashlan, "Physical layer security for $5 \mathrm{G}$ non-orthogonal multiple access in large-scale networks," in 2016 IEEE International Conference on Communications (ICC), Kuala Lumpur, May 2016, pp. 1-6.

[42] Y. Liu, Z. Qin, M. Elkashlan, Y. Gao, and F. Lajos Hanzo, "Enhancing the physical layer security of non-orthogonal multiple access in largescale networks," IEEE Trans. Wireless Commun., vol. 16, no. 3, pp. 16561672, Mar. 2017.

[43] H. Zhang, N. Yang, K. Long, M. Pan, G. K. Karagiannidis, and V. C. M. Leung. "Secure communications in NOMA system: Subcarrier assignment and power allocation". arXiv:1801.04441, Jan. 2018 , https://arxiv.org/abs/1801.04441

[44] G. Gomez, F. J. Martin-Vega, F. J. Lopez-Martinez, Y. Liu, and M. Elkashlan, "Uplink NOMA in large-scale systems: Coverage and physical layer security," arXiv:1709.04693, Sept. 2017, https://arxiv.org/abs/1709.04693.

[45] O. O. Koyluoglu, C. E. Koksal, and H. E. Gamal, "On secrecy capacity scaling in wireless networks," IEEE Trans. Inf. Theory, vol. 58, no. 5, pp. 3000-3015, May 2012

[46] X. Zhang, X. Zhou, and M. R. McKay, "Enhancing secrecy with multiantenna transmission in wireless ad hoc networks," IEEE Trans. Inf. Forensics Security, vol. 8, no. 11, pp. 1802-1814, Aug. 2013.

[47] R. L. Graham, D. E. Knuth, and O. Patashnik, Concrete Mathematics. New York, NY, USA: Addison-Wesley, 1989.

[48] Z. Chen, Z. Chi, Y. Li, and B. Vucetic, "Error performance of maximalratio combining with transmit antenna selection in flat Nakagami- $m$ fading channels," IEEE Trans. Wireless Commun., vol. 8, no. 1, pp. 424 431, Jan. 2009.

[49] J. Zhu, Y. Zou, G. Wang, Y.-D. Yao, and G. K. Karagiannidis, "On secrecy performance of antenna-selection-aided MIMO systems against eavesdropping," IEEE Trans. Veh. Technol., vol. 65, no. 1, pp. 214-225, Jan. 2016.

[50] H. Lei, C. Gao, I. S. Ansari, Y. Guo, Y. Zou, G. Pan, and K. Qaraqe, "Secrecy outage performance of transmit antenna selection for MIMO underlay cognitive radio systems over Nakagami- $m$ channels," IEEE Trans. Veh. Technol., vol. 66, no. 2, pp. 2237-2250, Mar. 2017.

[51] H. Lei, M. Xu, I. S. Ansari, G. Pan, K. A. Qaraqe, and M.-S. Alouini, "On secure underlay MIMO cognitive radio networks with energy harvesting and transmit antenna selection," IEEE Trans. Green Commun. Netw., vol. 1, no. 2, pp. 192-203, Jun. 2017.

[52] I. S. Gradshteyn and I. M. Ryzhik, Table of Integrals, Series and Products, 7th. San Diego,CA: Academic Press, 2007.

[53] M. Abramowitz and I. A. Stegun, Handbook of Mathematical Functions with Formulas, Graphs, and Mathematical Tables, 9th. New York, NY, USA: Dover Press, 1972.

[54] H. Lei, I. S. Ansari, G. Pan, B. Alomair, and M.-S. Alouini, "Secrecy capacity analysis over alpha - mu fading channels," IEEE Commun. Lett., vol. 21, no. 6, pp. 1445-1448, Jun. 2017.

[55] H. A. David and N. Nagaraja, Order Statistics, 3rd. Hoboken, NJ, USA: Wiley, 2003.

[56] F. Fang, H. Zhang, J. Cheng, S. Roy, and V. C. M. Leung, "Joint user scheduling and power allocation optimization for energy-efficient NOMA systems with imperfect CSI," IEEE J. Sel. Areas Commun., vol. 35, no. 12, pp. 2874-2885, Dec. 2017. 


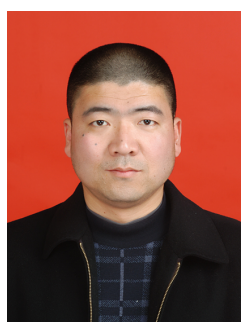

Hongjiang Lei (M'17) received the B.Sc. degree in Mechanical and Electrical Engineering from Shenyang Institute of Aeronautical Engineering, Shenyang, China, in 1998, the M.Sc. degree in Computer Application Technology from Southwest Jiaotong University, Chengdu, China, in 2004, and the Ph.D. degree in Instrument Science and Technology from Chongqing University, Chongqing, China, in 2015, respectively. In May 2004, he joined the School of Communication and Information Engineering of Chongqing University of Posts and Telecommunications (CQUPT), Chongqing, China, where he is currently an Associate Professor. Since Nov. 2016, he is also with Computer, Electrical, and Mathematical Science and Engineering (CEMSE) Division, King Abdullah University of Science and Technology (KAUST), Saudi Arabia, where he is a postdoc under the supervision of Prof. Mohamed-Slim Alouini. His research interest spans special topics in communications theory and signal processing, including physical layer security, and cognitive radio networks. He is a TPC Member of IEEE Globecom'17, IEEE Globecom'18, etc.. He has also served as a reviewer for major international journals, e.g., IEEE JSAC, IEEE TVT, IEEE TCOM, IEEE TWC, IEEE TIFS, IEEE WCL, IEEE CL, etc.

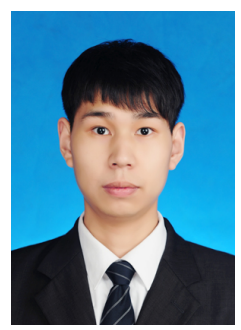

Jianming Zhang received the B.Sc. degree in Electrical Engineering from the Hebei University of Architecture, Zhangjiakou, China, in 2015. He is currently pursuing the M.Sc. degree in Information and Communication Engineering at Chongqing University of Posts and Telecommunications (CQUPT), Chongqing, China. His research interests include cognitive radio networks, physical layer security, and non-orthogonal multiple access.

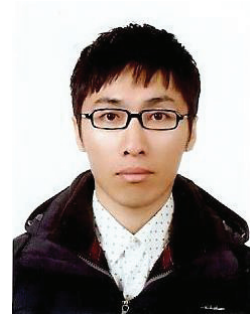

Ki-Hong Park (S'06-M'11) received the B.Sc. degree in electrical, electronic, and radio engineering from Korea University, Seoul, Korea, in 2005 and the M.S. and Ph.D. degrees from the School of Electrical Engineering, Korea University, Seoul, Korea, in 2011. Since April 2011, he has been a Postdoctoral Fellow of Electrical Engineering in the Division of Physical Science and Engineering, King Abdullah University of Science and Technology (KAUST), Thuwal, Saudi Arabia. His research interests are broad in communication theory and its application to the design and performance evaluation of wireless communication systems and networks. On-going research includes the application to MIMO diversity/beamforming systems, cooperative relaying systems, physical layer secrecy, and optical wireless communications.

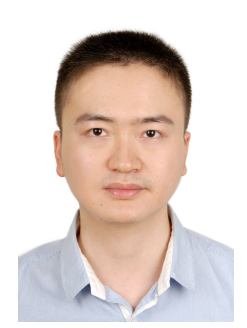

Xu Peng received the B.Eng. and Ph.D. degrees in electronic and information engineering from the University of Science and Technology of China, Anhui, China, in 2009 and 2014, respectively. Since 2014, he has been a Post-Doctoral with the Department of Electronic Engineering and Information Science, University of Science and Technology of China, Hefei, China. He is currently an Associate Professor with the School of Communication and Information Engineering, Chongqing University of Posts and Telecommunications, China. His current research interests include cooperative communications, information theory, information-theoretic secrecy, and 5G networks. He received the IEEE Wireless Communications Letters Exemplary Reviewer 2015.

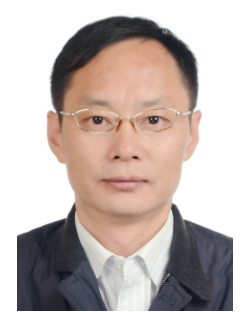

Zufan Zhang received his B.Eng. and M.Eng. degrees in 1995 and 2000, respectively from CQUPT, and his Ph.D. degree in Communications and Information Systems, University of Electronic Science and Technology of China (UESTC), Chengdu, China, in 2007. Prof. Zhang is currently a professor with School of Communication and Information Engineering, Chongqing University of Post and Telecommunications (CQUPT), Chongqing, China. $\mathrm{He}$ was a visiting professor at Centre for Wireless Communications (CWC), Oulu of University, Finland from Feb. 2011 to Jan. 2012. His current main research interest includes wireless communications, mobile social networks and machine learning.

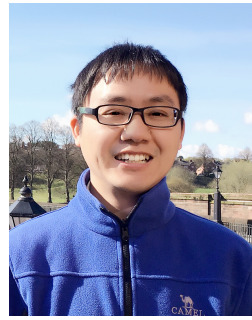

Gaofeng Pan (M'12) received his B.Sc in Communication Engineering from Zhengzhou University, Zhengzhou, China, in 2005, and the Ph.D. degree in Communication and Information Systems from Southwest Jiaotong University, Chengdu, China, in 2011. He was with The Ohio State University, Columbus, OH, USA, from Sept. 2009 to Sept. 2011 as a joint-trained $\mathrm{PhD}$ student under the supervision of Prof. Eylem Ekici. In May 2012, he joined the School of Electronic and Information Engineering, Southwest University, Chongqing, China, where he is currently an Associate Professor. Since Jan. 2016, he is also with School of Computing and Communications, Lancaster University, Lancaster, U.K., where he is a postdoc under the supervision of Prof. Zhiguo Ding. His research interest spans special topics in communications theory, signal processing and protocol design, including secure communications, CR communications and MAC protocols. From 2016 to now, he serves as a TPC Member of IEEE Globecom, IEEE ICC, and IEEE VTC etc. He has also served as a reviewer for major international journals, e.g., IEEE JSAC, IEEE TCOM, IEEE TWC, IEEE TSP, IEEE TVT, IEEE CL, IEEE WCL, etc.

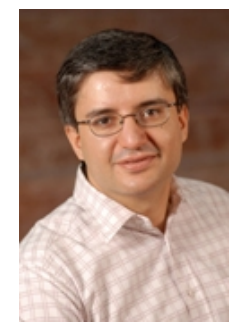

Mohamed-Slim Alouini (S'94-M'98-SM'03-F'09) was born in Tunis, Tunisia. He received the Ph.D. degree in Electrical Engineering from the California Institute of Technology (Caltech), Pasadena, CA, USA, in 1998. He served as a faculty member in the University of Minnesota, Minneapolis, MN, USA, then in the Texas A\&M University at Qatar, Education City, Doha, Qatar before joining King Abdullah University of Science and Technology (KAUST), Thuwal, Makkah Province, Saudi Arabia as a Professor of Electrical Engineering in 2009. His current research interests include the modeling, design, and performance analysis of wireless communication systems. 